\title{
Tryptase promotes breast cancer angiogenesis through PAR-2 mediated endothelial progenitor cell activation
}

\author{
NENG QIAN ${ }^{1}$, XIAOBO LI $^{2}$, XINHONG WANG $^{2}$, CHUNGEN WU $^{3}$, LIANHUA YIN $^{2}$ and XIULING ZHI ${ }^{3}$ \\ ${ }^{1}$ School of Basic Medicine, Shanghai University of Medicine and Health Science, Shanghai 201318; \\ ${ }^{2}$ Department of Physiology and Pathophysiology; ${ }^{3}$ Laboratory of Medical Molecular Biology, Training Center of \\ Medical Experiments, School of Basic Medical Sciences, Fudan University, Shanghai 200032, P.R. China
}

Received October 14, 2017; Accepted April 19, 2018

DOI: $10.3892 / \mathrm{ol} .2018 .8856$

\begin{abstract}
Mast cells have been demonstrated to accumulate around and within solid tumors of numerous types, and express a number of pro-angiogenic compounds, including tryptase. They may serve an early role in angiogenesis within developing tumors. In the present study, the role and mechanism of tryptase in the activation of endothelial progenitor cells (EPCs) in breast cancer angiogenesis were evaluated. Human umbilical cord blood EPCs were isolated and cultured. MB-MDA-231 breast cancer cells were then pretreated with tryptase, and the conditioned medium was collected. The effects of tryptase on the migratory and angiogenesis abilities of EPCs were determined using wound-healing and tube formation assays, respectively. The effect of tryptase on the proliferation of EPCs was detected using a Cell Counting Kit-8 assay. Alterations in proteinase activated receptor (PAR)-2, phosphorylated (p)-protein kinase B (AKT), p-extracellular signal-regulated kinase (p-ERK) and vascular endothelial growth factor receptor (VEGFR)-2 expression were analyzed, in tryptase or conditioned medium-treated EPCs, by western blot analysis and reverse transcription-quantitative polymerase chain reaction. It was confirmed that the EPCs expressed PAR-2; and that tryptase treatment promoted the migration and tube formation of EPCs. Treatment with a PAR-2 agonist had a similar effect to tryptase, whereas treatment with a tryptase inhibitor, APC366, or a PAR-2 inhibitor, SAM 11, inhibited the effect of tryptase treatment. Tryptase and PAR-2 agonists did not affect the rate of EPC proliferation. MB-MDA-231 cells also expressed PAR-2. Treatment with tryptase or conditioned medium increased the expression of PAR-2, p-AKT, p-ERK and VEGFR-2 in EPCs. In conclusion, tryptase activated
\end{abstract}

Correspondence to: Dr Xiuling Zhi, Laboratory of Medical Molecular Biology, Training Center of Medical Experiments, School of Basic Medical Sciences, Fudan University, 130 Dongan Road, Shanghai 200032, P.R. China

E-mail: zhixiuling@fudan.edu.cn

Key words: endothelial progenitor cell, tryptase, angiogenesis, proteinase activated receptor-2, breast cancer
EPCs via PAR-2-mediated AKT and ERK signaling pathway activation, thereby enhancing angiogenesis in breast cancer.

\section{Introduction}

Folkman developed the theory that the growth and metastasis of solid tumors are dependent on angiogenesis (1). In recent years, extensive research has demonstrated that the mobilization and differentiation of endothelial progenitor cells (EPCs) from the peripheral blood and bone marrow serves an important role in tumor angiogenesis (2).

Tumor cells and their microenvironment secrete multiple factors that induce EPC activation, including vascular endothelial growth factor (VEGF), angiopoietin, stromal cell-derived factor (SDF)-1 and matrix metallopeptidase (MMP)-9. Mast cells (MCs) accumulate around and within the microenvironments of numerous types of solid tumor (3). MC infiltration is associated with microvascular density and prior to the initiation of angiogenesis, increased numbers of MCs have been identified in various types of solid tumor, including breast cancer (4), gastric cancer (5) and lung cancer (6). MCs secrete a variety of angiogenic factors, including tryptase, chymase, tumor necrosis factor (TNF)- $\alpha$, interleukin (IL)-8, fibroblast growth factor (FGF) 2 and VEGF. Therefore, MCs serve a role in tumor angiogenesis, and are associated with tumor progression, metastasis and prognosis (7). Tryptase is the most abundant enzyme in MCs, and is stored as an active tetramer in complex with heparin in MC secretory granules. It has a variety of biological activities, including the ability to activate MCs, increase blood vessel permeability (8), induce the infiltration of inflammatory cells (9), and stimulate epithelial cell proliferation and the release of IL-8 (10). Previous studies have identified an association between the level of tryptase in the tumor microenvironment and angiogenesis in breast cancer (11).

Proteinase activated receptors (PARs) are members of the G-protein coupled receptor superfamily, and consist of four subtypes: PAR-1, PAR-2, PAR-3 and PAR-4. PAR-2 are activated by trypsin, tryptase, membrane-type serine protease-1, airway trypsin-like protease or coagulation factors VIIa and $\mathrm{Xa}$ (12). PAR-2 is expressed on the cell membrane of endothelial cells, vascular smooth muscle cells, fibroblasts, macrophages and MCs; its activation is associated with many inflammatory, 
respiratory, gastrointestinal, metabolic, cardiovascular, and neurological diseases, as well as cancers (13). Previous studies identified that PAR-2 expression was significantly higher in tumor tissue compared with in normal tissue, including in ovarian, colon and breast cancer, and that this may be associated with tumor angiogenesis $(11,14,15)$. Liu and Mueller (16) reported that $\mathrm{MDA}-\mathrm{MB}-231$ breast cancer cells exhibit a high expression of PAR-2, which, on activation, promotes the expression of VEGF via the extracellular signal-regulated kinase (ERK) 1/2 and p38 mitogen activated protein kinase (MAPK) signaling pathways. Using human umbilical cord blood-derived late-EPC, Smadja et al (17) identified that PAR-1 expression levels were similar in EPCs and human umbilical vein endothelial cells (HUVECs), and that treatment with PAR-1 tethered ligand peptides (SFLLRN), a PAR-1 and -2 activator, induced EPC proliferation, migration and differentiation. It was concluded from this data that the PAR-1 signaling pathway is involved in EPC-mediated angiogenesis, although the role of PAR-2 could not be excluded (17).

To the best of our knowledge, the expression and role of PAR-2 in EPC activation has not been previously reported. Therefore, the present study aimed to detect the effect of tryptase treatment on the activation of EPCs via PAR-2, which was previously demonstrated to promote angiogenesis in breast cancer (18).

\section{Materials and methods}

Tumor cells and reagents. MB-MDA-231 breast cancer cells, murine mammary carcinoma cell 4T1 and endothelioma cell bEnd. 3 were obtained from the American Type Culture Collection (Manassas, VA, USA). Endothelial cell growth medium (EGM) and SingleQuots combinatorial additive were purchased from Clonetics Corporation (San Diego, CA, USA). 1,1-dioctadecyl-3,3,3,3-tetramethylindocarbocyanine-labeled acetylated low density lipoprotein (Dil-Ac-LDL) was purchased from Thermo Fisher Scientific, Inc. (Molecular Probes; Waltham, MA, USA). The ReverTra Ace qPCR RT kit and SYBR Green Realtime PCR master mix were from Toyobo Life Science (Osaka, Japan). TRIzol was obtained from Thermo Fisher Scientific, Inc. (Invitrogen). High glucose Dulbecco's modified Eagle's medium (DMEM) and fetal bovine serum (FBS) were from Thermo Fisher Scientific, Inc. (Gibco). Fibronectin (Fn) and the PAR-2 agonist, 2-Furoyl LIGRLO-amide trifluoroacetate salt (2fLI), were from Merck KGaA (Sigma-Aldrich; Darmstadt, Germany). A selective inhibitor of MC tryptase, APC366 $\left(K_{\mathrm{i}}=7.1 \mu \mathrm{m}\right.$; cat. no. 178925-65-0) and a PAR-2-activating agonist peptide (SLIGRL-NH2; cat. no. 171436-38-7) were obtained from Tocris Bioscience (Bristol, UK). Unless otherwise indicated, purified tryptase with heparin $(1: 1, \mathrm{wt} / \mathrm{wt})$ was diluted with Minimum Essential medium (MEM) (Gibco; Thermo Fisher Scientific, Inc.) for use in the study. The western blot electrophoresis/transmembrane system was obtained from Bio-Rad Laboratories, Inc. (Hercules, CA, USA).

Culture and identification of EPCs. The protocol of the present study was approved by the ethical committee of the School of Basic Medical Sciences, Fudan University (Shanghai, China) and written informed consent was obtained from all patients.
Blood was collected from 3 patients (age range, 33-35 years) from the Obstetrics \& Gynecology Hospital of Fudan University from December 2010 to May 2017 respectively. As previously described (19), $20 \mathrm{ml}$ of fresh anticoagulant umbilical venous blood was collected. Mononuclear cells (MNCs) were isolated by density gradient centrifugation over Biocoll separating solution (Biochrom; Merck KGaA) at $500 \mathrm{x} g$ for $20 \mathrm{~min}$ at room temperature, and washed three times in PBS. MNCs were plated and $5 \times 10^{5}$ cells were seeded onto culture dishes coated with human Fn and cultured in EGM containing SingleQuots combinatorial additive at $37^{\circ} \mathrm{C}$ with $5 \% \mathrm{CO}_{2}$ in a humidified atmosphere. After 3 days, non-adherent EPCs were removed and fresh culture medium was added. The medium was replaced every third day, and the cells were passaged on day 14.

Cells from the third and fifth generations were observed and subsequently examined. In brief, cells were detached, blocked with $2 \%$ fetal calf serum (Invitrogen; Thermo Fisher Scientific, Inc.) at $4^{\circ} \mathrm{C}$ for $10 \mathrm{~min}$, washed and then incubated separately with phycoerythrin (PE)-conjugated VEGF receptor-2 (VEGFR-2; also known as KDR/Flk-1, cat. no. 130-100-308), FITC-conjugated cluster of differentiation (CD)34 (cat. no. 130-098-142) or PE-CD133 (cat. no. 130-098-872) antibodies (dilution 1:11; Miltenyi Biotec $\mathrm{GmbH}$, Bergisch Gladbach, Germany) at $4^{\circ} \mathrm{C}$ for $30 \mathrm{~min}$. Following the incubation, cells were washed with PBS containing $0.1 \%$ bovine serum albumin (Sigma-Aldrich; Merck KGaA) and analyzed by fluorescence-activated cell sorting with a FacsCalibur ${ }^{\mathrm{TM}}$ flow cytometer (BD Biosciences, Franklin Lakes, NJ, USA) using CellQuest software (version 5.1, BD Biosciences).

CD31 (cat. no. 550389; 1:50; BD Biosciences) was detected by immunocytochemical analysis. Isotype-identical antibodies (cat. no. 550878; 1:50; BD Biosciences) served as controls to exclude non-specific binding. The cytoplasm of the positively stained cells was brown, whereas negative cells remained colorless. To further verify that the cells were EPCs, the uptake of Dil-Ac-LDL, a function associated with endothelial cells, was assessed. Cells were incubated with $4 \mu \mathrm{g} / \mathrm{ml}$ Dil-Ac-LDL at $37^{\circ} \mathrm{C}$ for $2 \mathrm{~h}$, washed with PBS and fixed with $2 \%$ formaldehyde for $10 \mathrm{~min}$ at $37^{\circ} \mathrm{C}$. The incorporation of DiI-Ac-LDL was evaluated under an inverted fluorescence microscope (magnification, x200).

Proliferation, migration and lumen formation assay of EPCs. The proliferation of EPC was evaluated using a Cell Counting Kit (CCK)-8 assay (Dojindo Molecular Technologies, Inc., Kumamoto, Japan), performed according to the manufacturer's protocol. A total of $2 \times 10^{3}$ cells/well were incubated with $100 \mu \mathrm{l}$ EGM culture medium in 96-multiwell plates. Cells were cultured for $0,24,48$ or $72 \mathrm{~h}$ prior to the addition of $10 \mu \mathrm{lCCK}-8(5 \mathrm{mg} / \mathrm{ml})$ to the culture medium of each well. After a 1-h incubation at $37^{\circ} \mathrm{C}$, the absorbance at $450 \mathrm{~nm}$ of each well was measured with a Thermomax microplate reader (Molecular Devices, LLC, Sunnyvale, CA, USA). Each experiment was repeated three times, and the mean of the measurements was used.

An in vitro wound-healing assay was performed to measure cell migration, as previously described (20). Briefly, 5x10 EPCs were seeded into each well and incubated to form a confluent monolayer. Following scraping of the cell monolayer in a straight line with a p200 pipette tip to create a scratch, the 

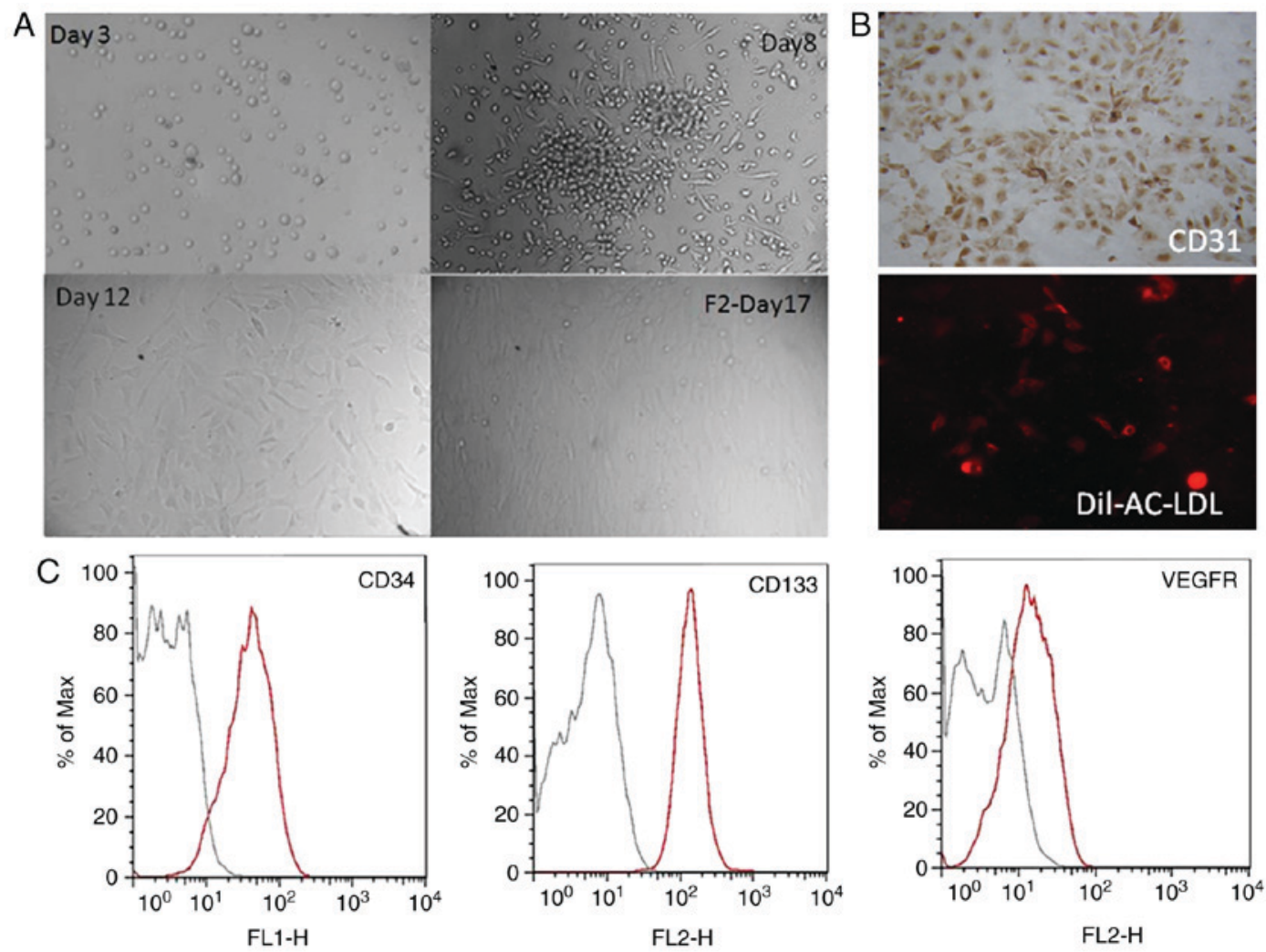

Figure 1. Culture and identification of EPCs. (A) EPCs were isolated and cultured from human umbilical cord blood (magnification, x100). (B) EPCs were identified by DiI-Ac-LDL uptake (red) and CD31 staining (brown; magnification, x200). (C) EPC purity was detected by fluorescence-activated cell sorting with CD34-FITC, CD133-PE or VEGFR-2-PE antibodies. Approximately $90 \%$ of the cells were identified as human umbilical cord endothelial progenitor cells (horizontal fluorescence intensity: Gray, negative control; red, positive). EPCs, endothelial progenitor cells; PE, phycoerythrin; VEGFR-2, vascular endothelial growth factor-2.

debris was removed and the edge of the scratch was smoothed by washing the cells once with $1 \mathrm{ml}$ growth medium (EGM). Then the growth medium was replaced with fresh EGM with no treatment, medium containing $1 \mathrm{nmol} / 1$ tryptase/heparin, $1 \mathrm{nmol} / 1$ tryptase/heparin with $25 \mu \mathrm{g} / \mathrm{ml}$ APC 366, $2.5 \mu \mathrm{g} / \mathrm{ml}$ 2fLI, $1 \mathrm{nmol} / 1$ tryptase/heparin with $0.2 \mu \mathrm{g} / \mathrm{ml} \mathrm{SAM} \mathrm{11,} \mathrm{or}$ $0.4 \mathrm{nmol} / \mathrm{l}$ heparin alone.

The tube formation ability of EPCs on the basement membrane was evaluated by plating cells on Matrigel, as previously described (21). Cells were divided into the same six treatment groups as described for the wound-healing assay.

Collection of conditioned media from MB-MDA-231 cells. The MB-MDA-231 cells $\left(3 \times 10^{5}\right.$ cells/well) were plated in 6 -well plates, and the cells were divided into three groups with serum-free DMEM with or without tryptase: $1 \mathrm{nmol} / \mathrm{l}$ Tryptase with $0.4 \mathrm{nmol} / 1$ heparin; $1 \mathrm{nmol} / 1$ Tryptase with $0.4 \mathrm{nmol} / 1 \mathrm{heparin}$ and $0.2 \mu \mathrm{g} / \mathrm{ml} \mathrm{SAM} 11$; or $0.4 \mathrm{nmol} / 1$ heparin only. After $48 \mathrm{~h}$, the culture medium from each group was centrifuged at $500 \times \mathrm{g}$ for $15 \mathrm{~min}$ at $4^{\circ} \mathrm{C}$, and the supernatants were filtered to create MB-MDA-231 cell-conditioned media. The conditioned media were then suitable for storage at $4^{\circ} \mathrm{C}$ for up to 3 months. EPCs were cultured in EGM plus the MB-MDA-231 cell-conditioned media (1:1, v/v) for $48 \mathrm{~h}$ before analysis.

Western blotting analysis. Cultured cells were washed with PBS twice and lysed in RIPA buffer (Beyotime Institute of
Biotechnology, Haimen, China) containing protease inhibitor and phosphatase inhibitor on ice. Supernatants were collected and the bicinchoninic acid method was used to determine the protein concentration. A total of $40 \mu \mathrm{g}$ protein/lane was subjected to $4-12 \%$ SDS-PAGE, and then transferred onto a polyvinylidene difluoride membrane. Membranes were blocked $2 \mathrm{~h}$ at room temperature in 5\% non-fat milk solution and then were incubated with Rabbit anti-PAR-2 (1:1,000; cat. no. 6976, Cell Signaling Technology, Inc., Danvers, MA, USA), VEGFR-2 (1:1,000; cat. no. 9698; Cell Signaling Technology, Inc.), phosphorylated (p)-protein kinase B (AKT; 1:2,000; cat. no. 4060; Cell Signaling Technology, Inc.) and p-ERK (1:1,000; cat. no. 4370; Cell Signaling Technology, Inc.) as the primary antibody, overnight at $4^{\circ} \mathrm{C}$, with agitation. Goat anti-rabbit horseradish peroxidase IgG H\&L (1:400, cat. no. ab97051; Abcam, Cambridge, UK) was used as the secondary antibody and was incubated at room temperature for $1 \mathrm{~h}$. GAPDH (cat. no. ab9485; 1:2,000) was used as a loading control. The blots were developed using an enhanced chemiluminescent autoradiography (Western BrightECL kit; cat. no. k-12045-D50; Advansta, Inc., Menlo Park, CA, USA). Blotting images were analyzed using ImageJ software v.1.6 (National Institutes of Health, Bethesda, MD, USA).

Reverse transcription-quantitative polymerase chain reaction (RT-qPCR) and semi-quantitative RT-PCR. TRIzol reagent was used for total RNA extraction according to the manufacturer's protocol, and 1,000 ng total RNA was used as a template for 

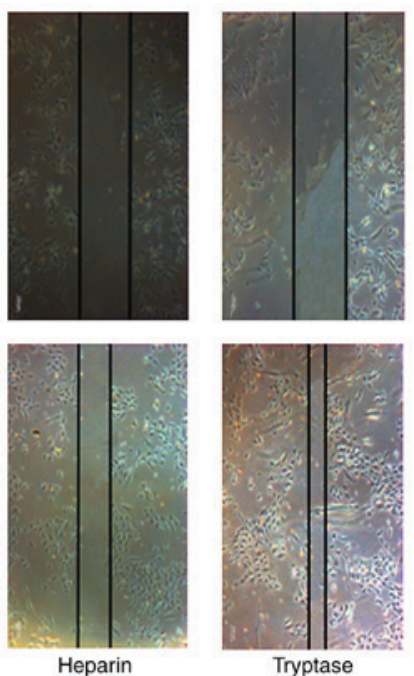

Tryptase
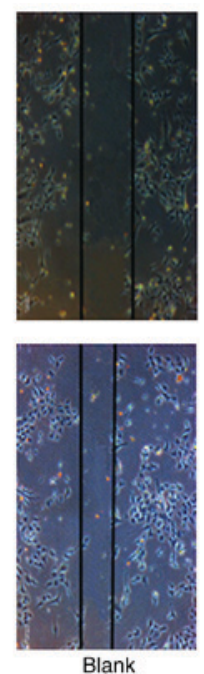
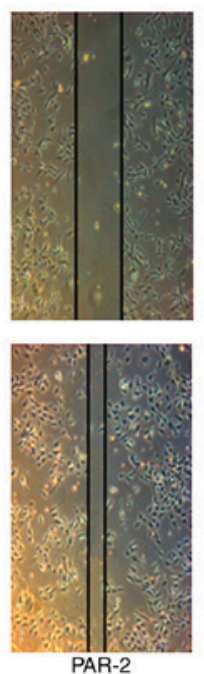
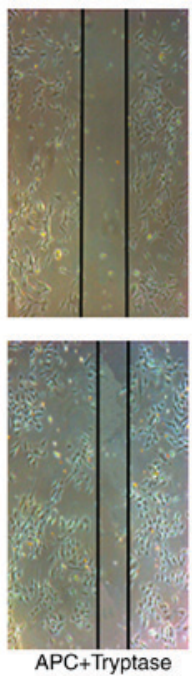
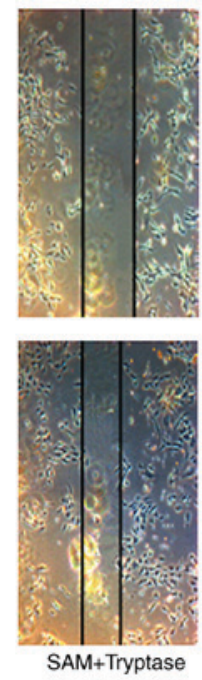

Figure 2. Analysis of EPC migration using wound-healing assays. Tryptase or PAR-2 agonists promoted the migration of EPCs, while treatment with tryptase inhibitor APC366 or PAR-2 inhibitor SAM 11 inhibited the effect of tryptase. Magnification, x100. EPCs, endothelial progenitor cells; PAR-2, proteinase activated receptor- 2 .

cDNA synthesis using the ReverTra Ace qPCR RT kit. qPCR was performed with a total reaction volume of $10 \mu$, including 10 ng of cDNA, $0.25 \mu \mathrm{M}$ forward and reverse primers and $5 \mu \mathrm{l}$ SYBR-Green qPCR master mix. The qPCR reaction conditions included initial denaturing at $94^{\circ} \mathrm{C}$ for $3 \mathrm{~min}, 30 \mathrm{sec}$ denaturing at $94^{\circ} \mathrm{C}, 30 \mathrm{sec}$ annealing at $59^{\circ} \mathrm{C}$ and $30 \mathrm{sec}$ extension at $72^{\circ} \mathrm{C}$ for 40 cycles, then a final incubation at $65^{\circ} \mathrm{C}$ for $5 \mathrm{~min}$. The amplified genes and the primers used were as follows: PAR-2 forward, TTCATGACCTGCCTCAGTGT and reverse, GTG ACCAGCAGAATCAGCAG (Gene ID: 2,150); VEGFR-2 forward, GTGATCGGAAATGACACTGGAG and reverse, CATGTTGGTCACTAACAGAAGCA; and GAPDH forward, ACAACTTTGGTATCGTGGAAGG and reverse, GCCATC ACGCCACAGTTTC. GAPDH was used as a loading control. Gene mRNA expression levels were analyzed using the $2^{-\Delta \Delta C q}$ method (22).

PAR-2 expression was also observed by semi-quantitative PCR in MB-MDA-231 breast cancer cells, murine mammary carcinoma cell 4T1, endothelioma cell bEnd.3 and EPC. The PCR reaction contained $1 \mu \mathrm{M}$ each of the forward and reverse primers, $10 \mu \mathrm{l}$ of $2 \mathrm{X}$ PCR master mix (Thermo Fisher Scientific, Inc.) and $1 \mu \mathrm{l}$ of cDNA in a total volume of $20 \mu \mathrm{l}$. The PCR products were visualized on a $2 \%$ agarose gel containing $5 \mu \mathrm{g} / \mathrm{ml}$ ethidium bromide.

Statistical analysis. Mean values were calculated from the data obtained from three or more separate experiments, and are presented as the mean \pm standard error of the mean. The significance of the differences between groups was estimated by one-way analysis of variance followed by a Student-Newman-Keuls test. SPSS version 19.0 (IBM Corp., Armonk, NY, USA) was used for analysis, and $\mathrm{P}<0.05$ was considered to indicate a statistically significant difference.

\section{Results}

Culture and identification of EPCs. Consistent with previous literature (20), freshly separated MNCs appeared small and rounded by $72 \mathrm{~h}$. When cultured on Fn-coated culture plates, from the third day, spindle-like cell morphology was visible at the edge of the cell clusters. By day 12, the adherent cells presented a typical cobblestone morphology (Fig. 1A). As presented in Fig. 1B, the EPCs expressed CD31 and had the ability to uptake Dil-Ac-LDL. CD133 is a marker for hematopoietic stem cells and EPCs, and its expression is gradually lost during the differentiation of EPCs into mature cells. As CD34, CD133 and VEGFR-2 are specific markers for EPCs, the expression of these markers was assessed by flow cytometry to further confirm the identity of the EPC. Approximately 90\% of the cells were CD34-, CD133- and VEGFR-2-positive (Fig. 1C).

Role of tryptase in promoting EPC migration. As demonstrated in Fig. 2, tryptase promoted EPC migration, and treatment with a PAR-2 agonist had a similar effect to tryptase. In addition, treatment with the tryptase inhibitor APC366 or the PAR-2 inhibitor SAM 11 reversed the effect of tryptase on EPC migration.

Tryptase promotes tube formation in EPCs. The formation of tube-like structures was more prominent in tryptase- or PAR-2 agonist-treated EPCs, whereas treatment with APC366 or SAM 11 decreased the tube formation ability of EPCs (Fig. 3).

Tryptase and PAR-2 agonists exhibit no effect on the proliferation of EPCs. A CCK-8 assay was used to analyze the proliferation rate of EPCs. It was identified that tryptase and PAR-2 agonists had no significant effect on the proliferation of EPCs at $0,24,48$ or $72 \mathrm{~h}$ (Fig. 4).

Effect of tryptase on the expression of PAR-2, $p$-AKT, $p$-ERK and VEGFR-2 in EPCs. PAR-2 was expressed in EPCs and MB-MDA-231 cells, as determined by RT-PCR detection (Fig. 5A). Similarly to positive control cells, PAR-2 expression was observed in murine mammary carcinoma cell 4T1 and endothelioma cell bEnd.3. The effect of tryptase on the mRNA 


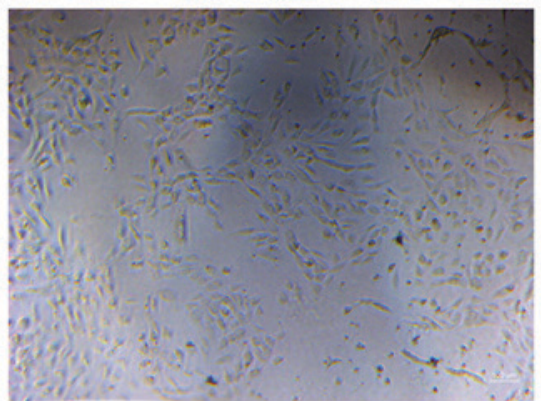

Heparin

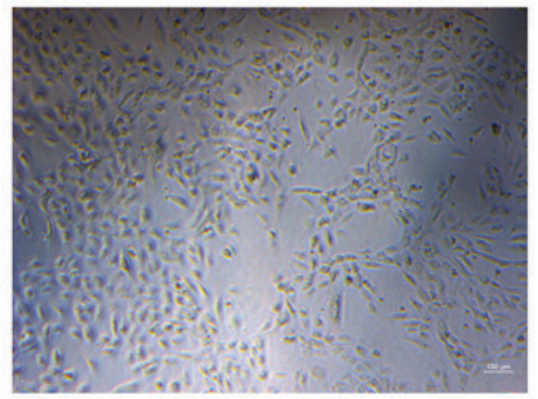

Blank

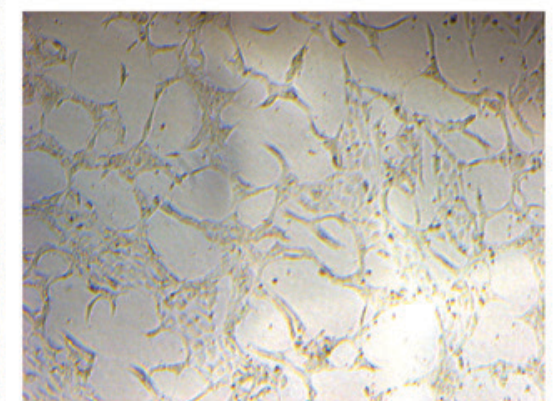

Tryptase

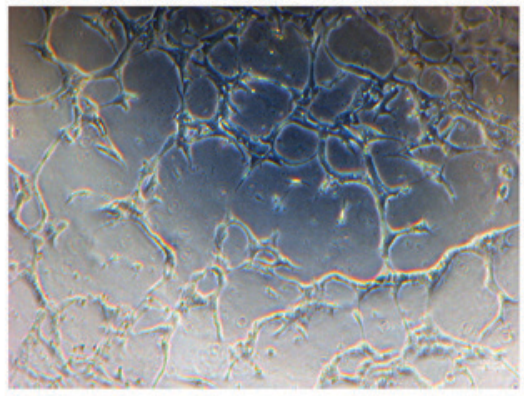

PAR-2

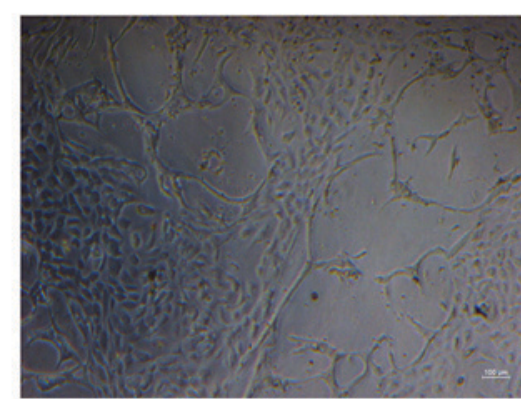

Tryptase+APC

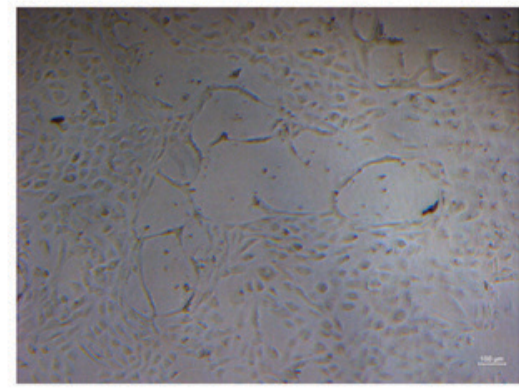

Tryptase+SAM

Figure 3. Tube formation ability of EPCs. Tryptase promoted the tube formation ability of EPCs; a PAR-2 agonist had a similar effect to tryptase, while tryptase inhibitor APC366 or PAR-2 inhibitor SAM 11 inhibited the effect of tryptase on tube formation Magnification, x100. EPCs, endothelial progenitor cells; PAR-2, proteinase activated receptor-2.

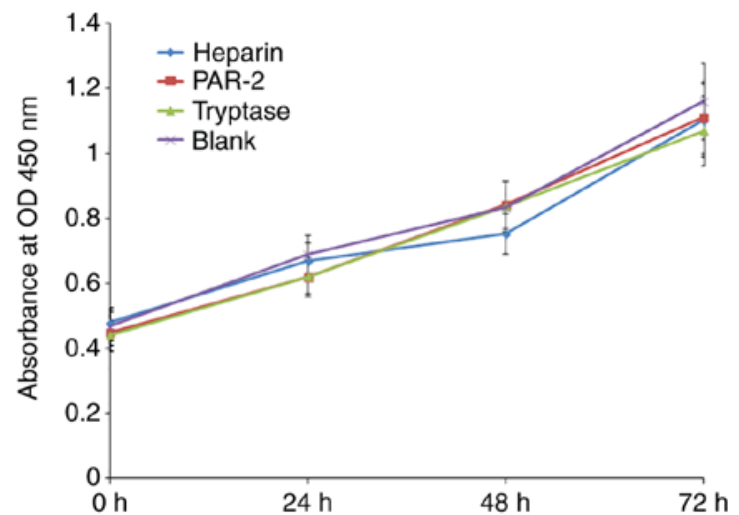

Figure 4. Proliferation of EPCs. The effect of tryptase on proliferation of EPCs was analyzed using a Cell Counting Kit-8 assay at 0,24, 48 and $72 \mathrm{~h}$. Tryptase and PAR-2 agonists had no evident effect on the proliferation of EPCs at any time point. EPCs, endothelial progenitor cells; PAR-2, proteinase activated receptor-2; OD, optical density.

expression of VEGFR-2 in EPCs was then analyzed using RT-qPCR. The data suggested that treatment with tryptase or a PAR-2 agonist significantly increased the VEGFR-2 mRNA level in EPCs compared with the blank control and herapin groups (all $\mathrm{P}<0.05)$; furthermore, treatment with APC 366 or SAM 11 reversed the effect of tryptase $(\mathrm{P}<0.05$; Fig. $5 \mathrm{~B})$. In addition, the expression of PAR-2, p-AKT, p-ERK and VEGFR-2 in EPCs was increased following treatment with tryptase or tryptase pretreated MB-MDA-231 cell-conditioned medium, as demonstrated with western blotting. These results suggest that tryptase may act directly on EPC through PAR-2/ERK signaling pathways (Fig. 5C), and on EPC indirectly through breast cancer cells MB-MDA-231 (Fig. 5D), but further studies are required to confirm this association. Thus,
MC tryptase may serve an important role in breast cancer angiogenesis by affecting the tumor microenvironment.

\section{Discussion}

There is increasing evidence to demonstrate thatEPCs are essential in the initial stages of carcinogenesis. Vajkoczy et al (23) reported that embryonic endothelial progenitor cells (eEPCs) isolated from stage E7.5 in mouse development at the onset of vasculogenesis retained their ability to contribute to tumor angiogenesis in the adult environment when systemically injected. eEPC homing was mediated by E- and P-selectin, and P-selectin glycoprotein ligand 1 . In a previous study in patients with tumor, the number and activation of EPCs were increased, and the number of circulating EPCs was demonstrated to be associated with the tumor volume (24). EPC activation, including migration, proliferation, homing and tube formation, controls the 'angiogenic switch' and contributes to the angiogenesis-mediated progression of micrometastases into potentially deadly macrometastases (25). The activation and recruitment of EPCs may be induced by angiogenic factors in the tumor microenvironment, including VEGF, angiopoietin, SDF-1, MMP-9 and platelet-derived growth factor (26). These factors are secreted by tumor cells and/or other cells, including tumor-associated macrophages or MCs, into the microenvironment.

Starkey et al (27) used genetically MC-deficient W/Wv mice to investigate the role of $\mathrm{MC}$ in tumor angiogenesis. It was reported that $\mathrm{W} / \mathrm{Wv}$ mice exhibited a lower tumor angiogenesis response and fewer lung metastases, while bone-marrow repair of the mast-cell deficiency restored the angiogenic response of $\mathrm{W} / \mathrm{Wv}$ mice, and restored the incidence of hematogenous metastases to approach that of $+/+$ 


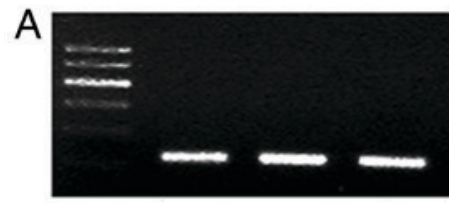

Marker 4T1 MDA231 bEnd3

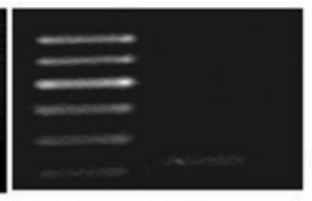

Marker EPC
$<125$ bp

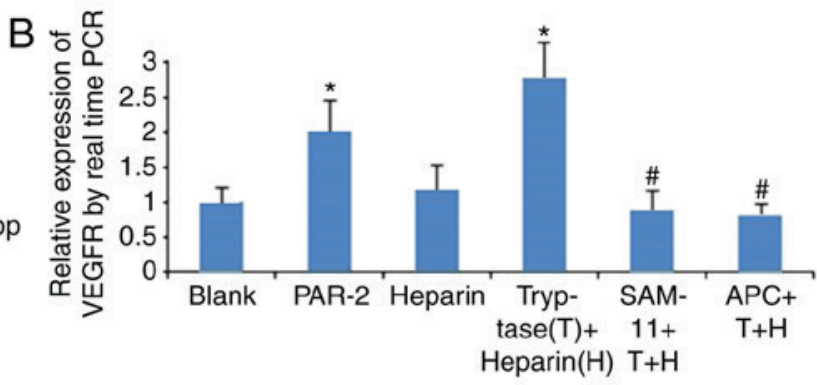

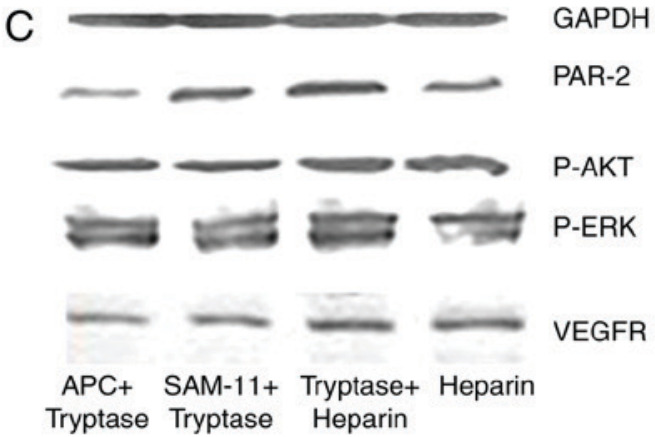

D

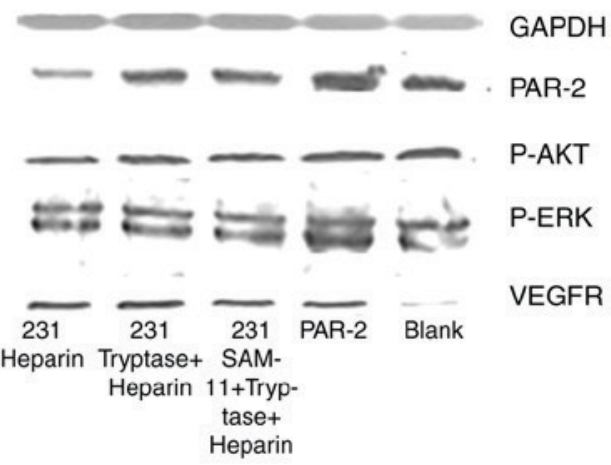

Figure 5. Effect of $\mathrm{T}$ on the expression of PAR-2, p-AKT, p-ERK and VEGFR-2 in EPCs. (A) EPCs and MB-MDA231 cells were confirmed to express PAR-2, as well as murine mammary carcinoma cell 4T1 and endothelioma cell bEnd.3. (B) mRNA level of VEGFR-2 in EPCs as determined by reverse transcription-quantitative PCR. Treatment with PAR2 or T increased the VEGFR-2 mRNA level, while the PAR-2 inhibitor SAM 11 or the tryptase inhibitor APC366 inhibited the effect of PAR2 and T, respectively. (C) T increased the expression of PAR-2, p-AKT, p-ERK and VEGFR-2 in EPCs, as determined by western blot analysis, whereas treatment with APC366 or SAM 11 inhibited the effect of tryptase. (D) Conditioned medium had the same effect as T. "P<0.05 vs. con, heparin, SAM and APC group, ${ }^{\#} \mathrm{P}<0.05$ vs. PAR2 and T groups. PAR-2, proteinase activated receptor-2; p-, phosphorylated; ERK, extracellular signal-regulated kinase; VEGFR-2, vascular endothelial growth factor-2; EPCs, endothelial progenitor cells; PAR2, PAR-2 agonist; T, tryptase; APC, APC366; con, control; AKT, protein kinase $\mathrm{B}$; PCR, polymerase chain reaction.

mice. These results indicate a role for MCs during tumor angiogenesis. In Kaposi's sarcoma, endometrial carcinoma, B-cell non-Hodgkin's lymphomas and breast cancer, the MC count was identified to be increased compared with normal tissue, and the microvascular density was positively associated with the MC number (18).

Tryptase is the most abundant enzyme in MCs, and serves an important role in a variety of biological activities, including inflammation and angiogenesis, in tumors and other diseases (28). Tryptase promotes angiogenesis, as demonstrated by Marech et al (29), induces lumen formation of endothelial cells (30), and degrades connective tissue matrix to provide adequate space for the formation of tumor blood vessels (31). In the present study, it was demonstrated that tryptase facilitated EPC migration and tube formation, but not proliferation, suggesting that tryptase and MCs may participate in tumor angiogenesis by activating EPCs.

PAR-2 is a receptor for thrombin, trypsin and tryptase that is expressed on cell membranes; it is associated with tumor cell adhesion, invasion and metastasis (32). Notably, these studies indicated that PAR-2 serves and essential role in cancer development, and it has indirect effects on angiogenesis in human vascular endothelial and tumor cell proliferation (33). Ge et al (34) observed that PAR-2 aggregated in the pseudopodia of metastatic breast cancer cells, and that its activation promoted tumor cell cytoskeletal remodeling and migration. PAR-2 silencing may inhibit the migration and invasion of the breast cancer cell lines MDA-MB-231 and BT549 (35). The EPCs derived from the bone marrow of mice were demonstrated to express PAR-2 (36), which is consistent with the data in the present study. The present study also demonstrated that the EPCs from human umbilical cord blood and MB-MDA-231 cells expressed PAR-2. The PAR-2 agonist mimicked the effect of tryptase on the proliferation, migration and tube formation of EPCs. In addition, tryptase and conditioned medium from tryptase-pretreated MBA-MD-231 cells increased the expression of PAR-2 in EPCs. The results of the current study indicate that tryptase may activate EPCs through PAR-2.

Previous studies have suggested that the effect of tryptase on the activation of endothelial cells may not be produced by the enzymatic cleavage of PAR-2, and that PAR-2 was genetically polymorphic (37-39). To determine whether PAR-2 mediates the role of tryptase in regulating the activation of EPCs, SAM 11 was used as an inhibitor of PAR-2 in the current study. SAM 11 is a monoclonal antibody against amino acids 37-50 in human PAR-2, which is the region at which PAR-2 activates peptides. Koo et al (40) reported that SAM 11 acts as an effective PAR-2 inhibitor. The results of the present study revealed that SAM 11 inhibited the effect of tryptase on EPC activation. In addition, treatment with tryptase or a PAR-2 agonist significantly promoted the expression of VEGFR-2 in EPCs, whereas treatment with SAM 11 or APC366, a tryptase inhibitor, significantly attenuated the effects of tryptase treatment. These data demonstrated that tryptase promoted EPC activation and angiogenesis via PAR-2.

The signaling pathways associated with the tryptase-mediated EPC activation in breast cancer angiogenesis by PAR-2 
were further investigated. The activation of PAR-2 may mediate several types of selective signal transduction in cells, particularly the amplifying cascade of the MAPK signaling pathway. Through the induction of the phosphorylation of MEK (41) and ERK $1 / 2$ (42), PAR-2 activation promotes cell hyperplasia, and subsequent structural changes at the tissue and organ levels. In human peripheral eosinophils, tryptase activates the MAPK/AP1 pathway, and promotes the synthesis and release of cytokines. Previous studies have identified that increased MC density is associated with the expression of p-AKT in human colon cancer tissue, indicating that the PI3K/AKT signaling pathway may be activated by tryptase (43). The PI3K/AKT signaling pathway activates a positive feedback loop to maintain the recruitment of inflammatory cells. In certain inflammatory and tumor environments, EPCs may be activated by the AKT and ERK signaling pathways (44). In the present study, treatment with tryptase, PAR-2 agonists and conditioned medium from tryptase-pretreated MBA-MD-231 human breast cancer cells promoted the expression of PAR-2, p-AKT, p-ERK and VEGFR-2 in EPCs, whereas treatment with APC366 and SAM 11 inhibited the effects of tryptase. These results confirmed that tryptase activated EPCs and promoted breast cancer angiogenesis via PAR-2-mediated AKT and ERK pathway activation.

In conclusion, tryptase may not only act directly on EPC activation, but also indirectly through breast tumor cells, to promote angiogenesis in breast cancer. This research provides a novel theoretical and molecular basis for anti-angiogenesis drug development.

\section{Acknowledgements}

The authors would like to thank Dr. Xianxian Sui and Ms. Fengdi Zhao for technical assistance.

\section{Funding}

The present study was supported by the National Natural Science Foundation of China (grant no. 81001170) and the Feed Fund of Shanghai University of Medicine and Health Sciences (grant no. HMSF-16-22-011).

\section{Availability of data and materials}

All data generated or analyzed during this study are included in this published article.

\section{Authors' contributions}

NQ performed PCR and Western blotting; XL, XW and CW performed the cell culture, wound healing and lumen formation assay; and LY and XZ performed EPC identification and data analysis and wrote the manuscript.

\section{Ethics approval and consent to participate}

The protocol of this study was approved by the Ethical Committee of The School of Basic Medical Sciences, Fudan University (Shanghai, China). Written informed consent was obtained from all patients.

\section{Consent for publication}

All authors have reviewed the manuscript and approved its submission for publication.

\section{Competing interests}

The authors declare no competing interests.

\section{References}

1. Folkman J: Antiangiogenesis in cancer therapy-endostatin and its mechanisms of action. Exp Cell Res 312: 594-607, 2006.

2. Roberts N, Jahangiri M and Xu Q: Progenitor cells in vascular disease. J Cell Mol Med 9: 583-591,2005.

3. Ribatti D, Ennas MG, Vacca A, Ferreli F, Nico B, Orru S and Sirigu P: Tumor vascularity and tryptase-positive mast cells correlate with a poor prognosis in melanoma. Eur J Clin Invest 33: 420-425, 2003.

4. Ribatti D, Finato N, Crivellato E, Guidolin D, Longo V, Mangieri D, Nico B, Vacca A and Beltrami CA: Angiogenesis and mast cells in human breast cancer sentinel lymph nodes with and without micrometastases. Histopathology 51: 837-842, 2007.

5. Mauro LV, Bellido M, Morandi A, Bonadeo F, Vaccaro C, Quintana GO, Pallotta MG, Lastiri J, Puricelli LI and De Cidre LL: Association between mast cells of different phenotypes and angiogenesis in colorectal cancer. Mol Med Rep 1: 895-902, 2008.

6. Micu GV, Stăniceanu F, Sticlaru LC, Popp CG, Bastian AE, Gramada E, Pop G, Mateescu RB, Rimba M, Archip B and Bleotu C: correlations between the density of tryptase positive mast cells (DMCT) and that of new blood vessels (CD105+) in patients with gastric cancer. Rom J Intern Med 54: 113-120, 2016.

7. Norrby K: Mast cells and angiogenesis. APMIS 110: 355-371, 2002.

8. He S and Walls AF: Human mast cell tryptase: A stimulus of microvascular leakage and mast cell activation. Eur J Pharmacol 328: 89-97, 1997.

9. He S, Peng Q and Walls AF: Potent induction of a neutrophil and eosinophil-rich infiltrate in vivo by human mast cell tryptase: Selective enhancement of eosinophil recruitment by histamine. J Immunol 159: 6216-6225, 1997.

10. Cairns JA and Walls AF: Mast cell tryptase is a mitogen for epithelial cells. Stimulation of IL-8 production and intercellular adhesion molecule-1 expression. J Immunol 156: 275-283, 1996.

11. Xiang M, Gu Y, Zhao F, Lu H, Chen S and Yin L: Mast cell tryptase promotes breast cancer migration and invasion. Oncol Rep 23: 615-619, 2010.

12. Ossovskaya VS and Bunnett NW: Protease-activated receptors: Contribution to physiology and disease. Physiol Rev 84: 579-621, 2004.

13. Yau MK, Liu L and Fairlie DP: Toward drugs for protease-activated receptor 2 (PAR2). J Med Chem 56: 7477-7497, 2013.

14. Jahan I, Fujimoto J, Alam SM, Sato E, Sakaguchi H and Tamaya T: Role of protease activated receptor- 2 in tumor advancement of ovarian cancers. Ann Oncol 18: 1506-1512, 2007.

15. Darmoul D, Gratio V, Devaud H and Laburthe M: Protease-activated receptor 2 in colon cancer: trypsin-induced MAPK phosphorylation and cell proliferation are mediated by epidermal growth factor receptor transactivation. J Biol Chem 279: 20927-20934, 2004.

16. Liu Y and Mueller BM: Protease-activated receptor-2 regulates vascular endothelial growth factor expression in MDA-MB-231 cells via MAPK pathways. Biochem Biophys Res Commun 344: 1263-1270, 2006

17. Smadja DM, Bièche I, Uzan G, Bompais H, Muller L, Boisson-Vidal C, Vidaud M, Aiach M and Gaussem P: PAR-1 activation on human late endothelial progenitor cells enhances angiogenesis in vitro with upregulation of the SDF-1/CXCR4 system. Arterioscler Thromb Vasc Biol 25: 2321-2327, 2005.

18. Ranieri G, Ammendola M, Patruno R, Celano G, Zito FA, Montemurro S, Rella A, Di Lecce V, Gadaleta CD, Battista De Sarro G and Ribatti D: Tryptase-positive mast cells correlate with angiogenesis in early breast cancer patients. Int J Oncol 35: $115-120,2009$. 
19. Eggermann J, Kliche S, Jarmy G, Hoffmann K, Mayr-Beyrle U, Debatin KM, Waltenberger J and Beltinger C: Endothelial progenitor cell culture and differentiation in vitro: A methodological comparison using human umbilical cord blood. Cardiovasc Res 58: 478-486, 2003.

20. Liang CC, Park AY and Guan JL: In vitro scratch assay: A convenient and inexpensive method for analysis of cell migration in vitro. Nat Protoc 2: 329-333, 2007.

21. Arnaoutova I, George J, Kleinman HK and Benton G: The endothelial cell tube formation assay on basement membrane turns 20: State of the science and the art. Angiogenesis 12: 267-274, 2009.

22. Livak KJ and Schmittgen TD: Analysis of relative gene expression data using real-time quantitative PCR and the 2(-Delta Delta C(T)) method. Methods 25: 402-408, 2001.

23. Vajkoczy P, Blum S, Lamparter M, Mailhammer R, Erber R, Engelhardt B, Vestweber D and Hatzopoulos AK: Multistep nature of microvascular recruitment of ex vivo-expanded embryonic endothelial progenitor cells during tumor angiogenesis. J Exp Med 197: 1755-1765, 2003.

24. Mancuso P, Burlini A, Pruneri G, Goldhirsch A, Martinelli G and Bertolini F: Resting and activated endothelial cells are increased in the peripheral blood of cancer patients. Blood 97: 3658-3661, 2001

25. Gao D, Nolan DJ, Mellick AS, Bambino K, McDonnell K and Mittal V: Endothelial progenitor cells control the angiogenic switch in mouse lung metastasis. Science 319: 195-198, 2008.

26. Li B, Sharpe EE, Maupin AB, Teleron AA, Pyle AL, Carmeliet P and Young PP: VEGF and PIGF promote adult vasculogenesis by enhancing EPC recruitment and vessel formation at the site of tumor neovascularization. FASEB J 20: 1495-1497, 2006.

27. Starkey JR, Crowle PK and Taubenberger S: Mast-cell-deficient $\mathrm{W} / \mathrm{Wv}$ mice exhibit a decreased rate of tumor angiogenesis. Int J Cancer 42: 48-52, 1988

28. Caughey GH: Mast cell tryptases and chymases in inflammation and host defense. Immunol Rev 217: 141-154, 2007.

29. Marech I, Ammendola M, Sacco R, Capriuolo GS, Patruno R, Rubini R, Luposella M, Zuccalà V, Savino E, Gadaleta CD, et al: Serum tryptase, mast cells positive to tryptase and microvascular density evaluation in early breast cancer patients: Possible translational significance. BMC Cancer 14: 534, 2014.

30. Blair RJ, Meng H, Marchese MJ, Ren S, Schwartz LB, Tonnesen MG and Gruber BL: Human mast cells stimulate vascular tube formation. Tryptase is a novel, potent angiogenic factor. J Clin Invest 99: 2691-2700, 1997.

31. Hiromatsu Y and Toda S: Mast cells and angiogenesis. Microsc Res Tech 60: 64-69, 2003.

32. Kawabata A: Gastrointestinal functions of proteinase-activated receptors. Life Sci 74: 247-254, 2003.

33. Ammendola M, Leporini C, Marech I, Gadaleta CD, Scognamillo G, Sacco R, Sammarco G, De Sarro G, Russo E and Ranieri G: Targeting mast cells tryptase in tumor microenvironment: A potential antiangiogenetic strategy. Biomed Res Int 2014: 154702, 2014.
34. Ge L, Shenoy SK, Lefkowitz RJ and DeFea K: Constitutive protease-activated receptor-2-mediated migration of MDA MB-231 breast cancer cells requires both beta-arrestin-1 and -2 . J Biol Chem 279: 55419-55424, 2004.

35. Wilson SR, Gallagher S, Warpeha K amd Hawthorne SJ: Amplification of MMP-2 and MMP-9 production by prostate cancer cell lines via activation of protease-activated receptors. Prostate 60: 168-174, 2004

36. Ma Y, Zhang B, Qian R, Lu C, Zhao F and Yin L: Tryptase activates PKB in inflammatory reaction in ECV304 cells. Biochim Biophys Acta 1763: 313-321, 2006.

37. Nonaka M, Pawankar R, Fukumoto A, Ogihara N, Sakanushi A and Yagi T: Induction of eotaxin production by interleukin-4, interleukin-13 and lipopolysaccharide by nasal fibroblasts. Clin Exp Allergy 34: 804-811, 2004.

38. Kohri K, Ueki IF and Nadel JA: Neutrophil elastase induces mucin production by ligand-dependent epidermal growth factor receptor activation. Am J Physiol Lung Cell Mol Physiol 283: L531-L540, 2002.

39. Compton SJ, McGuire JJ, Saifeddine M and Hollenberg MD: Restricted ability of human mast cell tryptase to activate proteinase-activated receptor-2 in rat aorta. Can J Physiol Pharmacol 80: 987-992, 2002.

40. Koo BH, Chung KH, Hwang KC and Kim DS: Factor Xa induces mitogenesis of coronary artery smooth muscle cell via activation of PAR-2. FEBS Lett 523: 85-89, 2002.

41. Yoshii M, Jikuhara A, Mori S, Iwagaki H, Takahashi HK, Nishibori M and Tanaka N: Mast cell tryptase stimulates DLD-1 carcinoma through prostaglandin- and MAP kinase-dependent manners. J Pharmacol Sci 98: 450-458, 2005.

42. Weidinger S, Mayerhofer A, Kunz L, Albrecht M, Sbornik M, Wunn E, Hollweck R, Ring J and Kohn FM: Tryptase inhibits motility of human spermatozoa mainly by activation of the mitogen-activated protein kinase pathway. Hum Reprod 20: 456-461, 2005.

43. Khan MW, Keshavarzian A, Gounaris E, Melson JE, Cheon EC, Blatner NR, Chen ZE, Tsai FN, Lee G, Ryu H, et al: PI3K/AKT signaling is essential for communication between tissue-infiltrating mast cells, macrophages, and epithelial cells in colitis-induced cancer. Clin Cancer Res 19: 2342-2354, 2013.

44. Wang JY, Lee YT, Chang PF and Chau LY: Hemin promotes proliferation and differentiation of endothelial progenitor cells via activation of AKT and ERK. J Cell Physiol 219: 617-625, 2009. 